\title{
Variables de personalidad en estudiantes universitarios en función de la rama de conocimiento*
}

\author{
Cristian CAStellano Badenas \\ al107103@alumail.uji.es \\ ELEnA FERMOSELLe Esclapez \\ al076962@alumail.uji.es \\ JORGE JAVIER OSMA LÓPEZ \\ osma@psb.uji.es \\ Elena CREspo Delgado \\ screspo@psb.uji.es
}

\section{Resumen}

Introducción: El Espacio Europeo de Educación Superior (EEES) proporciona un marco perfecto en el que explorar algunas de las variables de la personalidad de los estudiantes universitarios. Los rasgos de la personalidad pueden convertirse en grandes predictores de comportamientos por lo que se deben analizar aquellos rasgos, que pueden facilitar un desempeño profesional efectivo.

Objetivo: Analizar determinadas características psicológicas de una muestra de estudiantes de la Universidad Jaume I (UJI) con el objetivo de conocer el perfil de personalidad de los estudiantes universitarios, así como averiguar si existen diferencias significativas según la rama de conocimiento.

Método: La evaluación para analizar dichas variables se hizo a través del Inventario de Personalidad NEO Revisado (NEO-PI-R). Se contactó con profesores de las distintas facultades de la UJI para obtener su colaboración. EI NEO-PI-R se administró a una muestra de 107 estudiantes universitarios.

Resultados: Las medias obtenidas en los factores y facetas de personalidad, indican que el perfil de personalidad de nuestra muestra es: Alto Neuroticismo, Media Extraversión y Apertura, y Baja Amabilidad y Responsabilidad. Se han obtenido diferencias significativas a favor de la FCHS en los factores Apertura y Amabilidad, en las facetas Sentimientos y Valores del factor Apertura y en las facetas Franqueza y Modestia del factor Amabilidad.

Conclusiones: Los estudiantes de la FCHS obtienen puntuaciones con diferencias significativas respecto al resto de facultades en algunas características de personalidad relacionadas con la competencia social. Será necesario establecer estrategias en

\footnotetext{
* Proyecto financiado a través de la Convocatoria 2010-2011 de Ayudas a Proyectos de Investigación y Formación OPI-UJI: Migració $i$ Interculturalitat de la Oficina de Cooperació al Desenvolupament i Solidaritat (OCDS) y el Observatori Permanent d'Inmigració (OPI) de la Universitat Jaume I.
} 
la educación superior para garantizar, en todos los estudiantes, la adquisición de competencias sociales para su futura inserción laboral.

Palabras clave: personalidad, NEO PI-R, Espacio Europeo de Educación Superior, estudiantes universitarios, Big Five, competencia social.

\section{Abstract}

Introduction: The European Space of Higher Education (ESHE) provides a perfect setting to explore some of the university students' personality variables. Personality characteristic can become great predictors of behaviours, that is the reason why those characteristic which can represent necessary aspects and skills for an effective and enterprising professional performance.

Goal: Analysing particular psychological characteristic from a sample of the university community originally from UJI with the intention of checking university students' personality profiles and verifying if there are significant differences from the different schools within the university.

Method: The evaluation for analysing the aforementioned variables was devised through the Revised NEO Personality Inventory (R-NEO-PI). We were in contact with teachers from the different schools at UJI in order to ensure their cooperation. The R-NEO-PI was distributed to a sample of 107 university students.

Results: The averages which have been obtained in personality factors and aspects, show that the personality profile from our sample is: High Neuroticism, Medium Extraversion and Openness and Low Agreeableness and Conscientiousness. We have obtained significant differences in favour of the School of Human and Social Sciences with regard to Openness and Agreeableness factors, Feelings and Values facets from Openness factor and Frankness and Modesty facets from Agreeableness factor.

Conclusions: The students from the School of Human and Social Sciences got scores with significant differences in relation to the rest of the schools in some personality characteristic linked to social competence. It will be necessary establishing strategies in higher education in order to guarantee, to all students, the acquisition of social skills for their future labor entry.

Keywords: Personality, NEO PI-R, ESHE, predictors, European Space of Higher Education, university students', Big Five,

\section{Introducción}

El Espacio Europeo de Educación Superior (EEES) es un plan de organización educativo que tiene como finalidad armonizar las enseñanzas universitarias en más de 40 países de todo el mundo. La educación superior debería jugar un papel esencial en la promoción de la cohesión social, en la reducción de las desigualdades y en la elevación del nivel del conocimiento, destrezas y competencias en el seno de la sociedad. La política debe, por tanto, aspirar a maximizar el potencial de los individuos en términos de su desarrollo personal y su contribución a la sostenibilidad democrática y la sociedad del conocimiento (Comunicado de Londres, 
2007). El Proceso de Bolonia ha propiciado a lo largo de la Unión Europea, nuevos y diferentes enfoques en la estructura de los sistemas educativos y en sus contenidos, objetivos y características. Entre algunas de las prioridades del EEES para la próxima década está la búsqueda de la excelencia frente a las cambiantes demandas de la sociedad en evolución (Conferencia de European Ministers Responsible for Higher Education, Lauven, 2009). Existen directrices específicas para alcanzar muchos de los objetivos que conforman los principios del Proceso de Bolonia, como los implicados en la dimensión social, el aprendizaje a lo largo de la vida, la excelencia en la gestión de la calidad del EEES dentro del sistema educativo español y la movilidad de los estudiantes.

Existen distintos estudios que ponen de manifiesto la importancia de la evaluación de las características de personalidad de los estudiantes con el objetivo de identificar y describir aquellas características facilitadoras del intercambio y adaptación intercultural y, por el contrario, aquellas características que dificultarían este proceso. Entre las primeras, se identifica la Extraversión y Apertura a la experiencia y, entre las segundas, el Neuroticismo. Los distintos estudios utilizan el NEO-PI-R como medida de evaluación de la personalidad y las evaluaciones las han realizado en muestras de estudiantes que deben desplazarse a otros países para realizar estudios universitarios (Chataway y Berry, 1989; Furukawa y Shibayama, 1993, 1994; Mak y Tran, 2001).

La sociedad moderna delega en la educación superior la función de desarrollar en los estudiantes las competencias que les permitan actuar de manera eficaz en dicha sociedad (Rodríguez-Izquierdo, 2008). De este modo, el EEES proporciona un marco idóneo en el que explorar la línea base de algunas de las competencias sociales básicas de los estudiantes, junto con sus variables de personalidad, con el objetivo de vislumbrar nuevas disciplinas, olvidadas hasta ahora, hacia las que dirigir futuros esfuerzos para mejorar y entrenar habilidades tan importantes en los estudiantes, como la tolerancia, el respeto hacia los demás o la curiosidad por lo diferente.

A través de este nuevo sistema educativo, y su línea de actuación enfocada desde una dimensión más social y personal, se puede hacer hincapié y dar prioridad al crecimiento personal de los propios estudiantes, al desarrollo y/o mejora de sus habilidades y recursos, y en definitiva a acercar al éxito en el futuro profesional de los estudiantes, ofreciéndoles las competencias adecuadas para adaptarse al mundo laboral al que tendrán que hacer frente una vez terminen su formación.

\section{Método}

\section{Objetivo}

El objetivo principal de este proyecto es revisar y analizar algunas características psicológicas de una muestra de estudiantes universitarios, con el fin de conocer, por una parte, qué dimensiones de personalidad predominan en la muestra del estudio y, por otra parte, averiguar si las dimensiones encontradas están relacionadas con las competencias personales y sociales. Por último, nos interesa averiguar si existen diferencias significativas en dimensiones de personalidad entre los estudiantes de distintas ramas de conocimiento. Para ello nos hemos centrado en el análisis de las dimensiones de la personalidad: Neuroticismo, Extraversión, Apertura, Amabilidad y Responsabilidad y sus respectivas facetas. En función de los resultados obtenidos se pueden mejorar y potenciar aquellas competencias y habilidades necesarias para el éxito de los futuros profesionales, aplicando diferentes técnicas en la metodología y docencia según la facultad o rama de conocimiento. 


\section{Procedimiento}

Se contactó con profesores de las distintas facultades de la UJI (Facultat de Ciències Humanes i Socials, Facultat de Ciències Jurídiques i Econòmiques i L'Escola Superior de Tecnologia i Ciències Experimentals) para obtener su colaboración en la tarea de distribución de los protocolos de evaluación del proyecto, en las diferentes asignaturas en las que impartían docencia en el curso académico 2009/2010.

Una vez establecidos los contactos de cooperación con profesores de la UJI, se les facilitaron los protocolos de evaluación para los alumnos/as de sus clases que desearan participar voluntariamente, dándoles una hoja de comunicación que describía cuestiones relacionadas con la confidencialidad de los datos, fechas de entrega de los instrumentos debidamente cumplimentados, lugar, etc. El objetivo de esta hoja de comunicación fue que todos los docentes ofrecieran la misma información a los/las estudiantes participantes disminuyendo así la posibilidad de sesgar sus respuestas.

Finalmente, los propios profesores colaboradores establecieron diferentes fechas a lo largo del curso para que los estudiantes entregaran los protocolos de evaluación cumplimentados. Una vez recogidos, el equipo investigador de este proyecto se encargó de su corrección.

\section{Muestra}

Se consideró una única muestra bajo estudio compuesta por estudiantes universitarios de la UJI de Castellón, que estuvieran matriculados en cualquiera de las titulaciones de grado o posgrado ofertadas durante el curso académico 2009/2010. La muestra final del estudio estuvo formada por 107 participantes, 41 mujeres y 66 hombres (figura 1) todos ellos estudiantes de grado o máster de la UJI de Castellón. La media de edad es de 24,26 años (DT: 4,95). En el momento de realizar la evaluación, el 52,3 \% realiza sus estudios en la Facultat de Ciències Humanes i Socials, el 20,6 \% en la Facultat de Ciències Juridiques i Econòmiques y el 26,2 \% en l'Escola Superior de Tecnologia i Ciències Experimentals (figura 2).

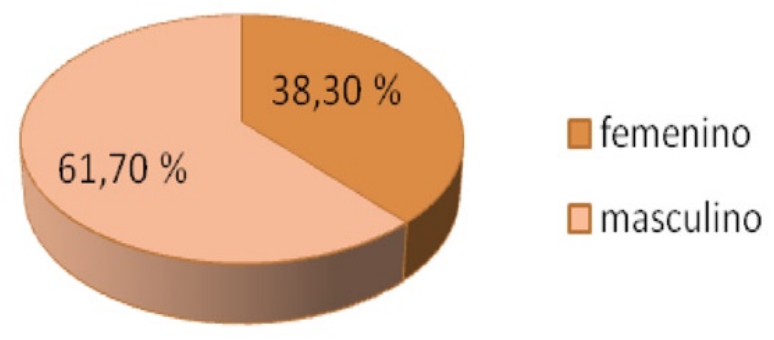

Figura 1. Distribución de la muestra de participantes por sexos 


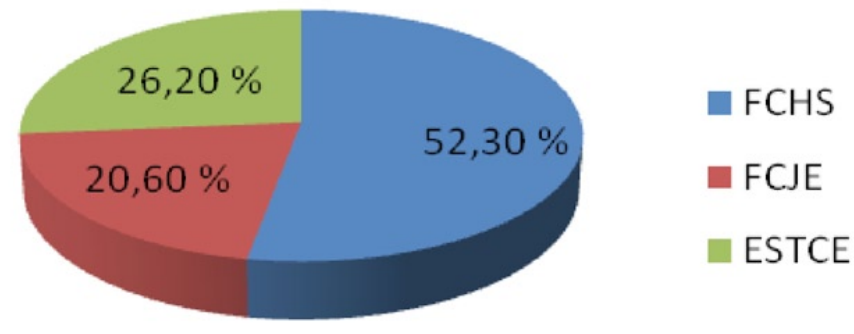

Figura 2. Distribución de la muestra de participantes por facultad

\section{Medida utilizada}

El instrumento de evaluación que fue utilizado para el análisis de las variables de personalidad de los estudiantes universitarios fue el Inventario de Personalidad NEO Revisado, NEO-PI-R (Costa y McCrae, 1992, validado en población española por Cordero, Pamos y Seisdedos, TEA Ediciones). EI NEO-PI-R es un inventario que evalúa la personalidad desde un enfoque dimensional mediante 240 ítems. La persona responde al inventario mediante una escala de 5 puntos que va desde 1 (en total desacuerdo) a 5 (totalmente de acuerdo). EI NEO-PI-R ofrece un perfil de la personalidad basado en las 5 dimensiones o factores básicos y sus respectivas facetas. Los factores que describe el inventario NEO-PI-R son: Neuroticismo, Extraversión, Apertura, Amabilidad y Responsabilidad. El manual del inventario NEO-PI-R recoge los numerosos estudios que han mostrado evidencia de las buenas propiedades psicométricas de este instrumento. Respecto a la fiabilidad se obtienen coeficientes alpha de entre 0.86 y 0.92 ; la fiabilidad test-retest también se ha mostrado satisfactoria con índices en las facetas que varían entre 0.69 y 0.92 y entre 0.86 y 0.91 en los factores. EI NEO-PI-R presenta una buena validez de constructo, convergente y discriminante. A continuación detallaremos únicamente los datos sobre propiedades psicométricas en población española de los principales factores. Respecto a la fiabilidad se obtienen altos índices de consistencia interna en Neuroticismo (0.90), Extraversión (0.84), Apertura (0.82), Amabilidad (0.83) y Responsabilidad (0.88). Respecto a la validez de constructo la estructura del inventario fue muy similar a la original.

\section{Análisis estadísticos}

Para analizar los datos obtenidos y poner a prueba las hipótesis planteadas, se utilizó estadística paramétrica mediante el paquete estadístico para las ciencias sociales (SPSS) en su versión 13.0 (SPSS 13.0, SPSS Inc, Chicago, IL). Concretamente, para la comparación de medias teniendo en cuenta determinadas variables y grupos, se llevó a cabo la prueba t de Student para muestras independientes y se consideraron los valores a partir de $p<0.05$ como criterio para establecer las diferencias estadísticamente significativas. Para la comparación, teniendo en cuenta las tres facultades universitarias, se empleó el análisis ANOVA de un factor. 


\section{Resultados}

Las medias obtenidas en los factores y facetas de personalidad evaluadas con el Inventario NEO-PI-R indican que, si tenemos en cuenta los cinco grandes factores, el perfil de personalidad de nuestra muestra de estudiantes universitarios es: Alto Neuroticismo, Media Extraversión y Apertura, y Baja Amabilidad y Responsabilidad.

Si nos centramos en analizar dimensión a dimensión, se pueden observar (tabla 1) las puntuaciones altas obtenidas en el Neuroticismo, por lo que la muestra de estudiantes se caracteriza, en términos generales, por el desajuste/inestabilidad emocional, una tendencia hacia los sentimientos negativos (como el ridículo y los sentimientos de inferioridad), un nivel bajo respecto a las competencias para hacer frente al estrés y un menor control de los impulsos. Si observamos las facetas de esta dimensión de la personalidad, se pueden destacar las altas puntuaciones en las facetas Ansiedad Social, Impulsividad y Vulnerabilidad.

Tabla 1

Resultados de la dimensión Neuroticismo y sus respectivas facetas

\begin{tabular}{lcc}
\hline \multicolumn{1}{c}{ NEO-PI-R } & MEDIAS (DT) & INTERPRETACIÓN \\
\hline Neuroticismo & $58,13(7,98)$ & Alto \\
Ansiedad & $55,29(9,09)$ & Alto \\
Hostilidad & $56,22(10,14)$ & Alto \\
Depresión & $55,22(10,21)$ & Alto \\
Ansiedad Social & $57,16(9,24)$ & Alto \\
Impulsividad & $57,79(8,48)$ & Alto \\
Vulnerabilidad & $58,64(8,52)$ & Alto \\
\hline
\end{tabular}

En referencia a las dimensiones Extraversión y Apertura, tablas 2 y 3 respectivamente, las puntuaciones obtenidas han sido medias, excepto en las facetas Asertividad, en las que se han obtenido puntuaciones bajas, denotando el déficit en las habilidades interpersonales y de liderazgo, y en la faceta Fantasía, donde las puntuaciones han sido superiores a la media, revelando el alto uso de la imaginación y la creatividad en los estudiantes de la Universidad.

Tabla 2

Resultados de la dimensión Extraversión y sus respectivas facetas

\begin{tabular}{lcc}
\hline \multicolumn{1}{c}{ NEO-PI-R } & MEDIAS $(\mathrm{DT})$ & INTERPRETACIÓN \\
\hline Extraversión & $47,50(9,32)$ & Medio \\
Cordialidad & $46,92(9,76)$ & Medio \\
Gregarismo & $47,00(10,27)$ & Medio \\
Asertividad & $44,76(9,69)$ & Bajo \\
Actividad & $47,25(9,85)$ & Medio \\
Búsqueda Emociones & $54,89(9,01)$ & Medio \\
Emociones Positivas & $50,29(9,97)$ & Medio \\
\hline
\end{tabular}


Tabla 3

Resultados de la dimensión Apertura y sus respectivas facetas

\begin{tabular}{lcc}
\hline \multicolumn{1}{c}{ NEO-PI-R } & MEDIAS $(\mathrm{DT})$ & INTERPRETACIÓN \\
\hline Apertura & $50,15(9,10)$ & Medio \\
Fantasía & $56,20(9,32)$ & Alto \\
Estética & $47,26(8,96)$ & Medio \\
Sentimientos & $51,21(9,09)$ & Medio \\
Acciones & $47,61(9,54)$ & Medio \\
Ideas & $48,24(9,13)$ & Medio \\
Valores & $51,33(10,54)$ & Medio \\
\hline
\end{tabular}

Por último, en las dimensiones Amabilidad y Responsabilidad, las puntuaciones en general han sido bajas. En la tabla 4 se pueden observar las bajas puntuaciones en Amabilidad, en la que se pueden destacar los niveles bajos en Sensibilidad, Actitud Conciliadora y Altruismo, indican una actitud escéptica y crítica de los estudiantes, una tendencia hacia la oposición de cooperar con los demás y pensamientos de suspicacia respecto a las intenciones de los demás.

Tabla 4

Resultados de la dimensión Amabilidad y sus respectivas facetas

\begin{tabular}{lcc}
\hline \multicolumn{1}{c}{ NEO-PI-R } & MEDIAS (DT) & INTERPRETACIÓN \\
\hline Amabilidad & $44,81(8,89)$ & Bajo \\
Confianza & $45,03(8,95)$ & Bajo \\
Franqueza & $49,20(9,09)$ & Medio \\
Altruismo & $44,92(9,98)$ & Bajo \\
Actitud Conciliadora & $44,05(9,02)$ & Bajo \\
Modestia & $50,38(8,47)$ & Medio \\
Sensibilidad & $44,32(8,20)$ & Bajo \\
\hline
\end{tabular}

Acerca de la dimensión Responsabilidad (tabla 5), todas las puntuaciones han resultado ser inferiores a la media, reflejando una muestra de estudiantes universitarios con dificultades en la organización y planificación de las tareas. Además, los niveles muy bajos en las facetas Autodisciplina y Logro, hacen referencia, respectivamente, a la desmotivación y dilatación del inicio de sus quehaceres, desanimándose fácilmente y con fácil deseo de abandonar, y a la falta de recursos para luchar por sus propios objetivos y aspiraciones, mostrando una actitud perezosa. 
Tabla 5

Resultados de la dimensión Responsabilidad y sus respectivas facetas

\begin{tabular}{lcc}
\hline \multicolumn{1}{c}{ NEO-PI-R } & MEDIAS $(\mathrm{DT})$ & INTERPRETACIÓN \\
\hline Amabilidad & $44,81(8,89)$ & Bajo \\
Confianza & $45,03(8,95)$ & Bajo \\
Franqueza & $49,20(9,09)$ & Medio \\
Altruismo & $44,92(9,98)$ & Bajo \\
Actitud Conciliadora & $44,05(9,02)$ & Bajo \\
Modestia & $50,38(8,47)$ & Medio \\
Sensibilidad & $44,32(8,20)$ & Bajo \\
\hline
\end{tabular}

Resultados según la rama de conocimiento

Por lo que respecta a los factores y facetas de personalidad evaluadas con el Inventario NEO-PI-R, y teniendo en cuenta a que facultad pertenece la muestra de estudiantes, se han hallado diferencias con significación estadística a favor de la FCHS en los factores Apertura $(p<0.05)$ y Amabilidad $(p<0.05)$. Por una parte, las personas con altas puntuaciones en Apertura están interesadas tanto por el mundo exterior como por el interior y sus vidas están enriquecidas por la experiencia. Desean considerar nuevas ideas y valores no convencionales, y experimentan tanto las emociones positivas como las negativas de manera más profunda que los individuos que son más cerrados. Por otra parte, la persona amable es fundamentalmente altruista. Simpatiza con los demás, está dispuesta a ayudarles y cree que los otros se sienten igualmente satisfechos de hacer lo mismo, considerando a la gente honesta y merecedora de su confianza.

Tabla 6

Medias y desviación típica de las medidas que han obtenido significación estadística en la comparación de medias (ANOVA) entre las 3 facultades universitarias

\begin{tabular}{lcccccc}
\hline \multicolumn{1}{c}{ NEO-PI-R } & FCHS & ESTCE & FCJE & DF & F & P \\
& M (DT) & M (DT) & M (DT) & & & \\
\hline Apertura & $52,14(8,86)$ & $48,89(9,08)$ & $46,83(8,84)$ & 2 & 3,277 & 0,042 \\
Amabilidad & $46,57(9,55)$ & $44,39(8,22)$ & $41,04(6,85)$ & 2 & 3.331 & 0,040 \\
Sentimientos & $53,19(9,46)$ & $49,96(7,50)$ & $47,91(9,03)$ & 2 & 3.247 & 0,043 \\
Valores & $54,55(9,58)$ & $49,42(10,81)$ & $45,78(9,97)$ & 2 & 6.958 & 0,001 \\
Franqueza & $51,39(10,56)$ & $47,35(6,63)$ & $46,13(6,20)$ & 2 & 3.699 & 0,028 \\
Modestia & $51,55(8,89)$ & $51,53(7,83)$ & $46,13(6,97)$ & 2 & 3.893 & 0,023 \\
\hline
\end{tabular}

* FCHS: Facultad de Ciencias Humanas y Sociales; ESTCE: Escuela Superior de Tecnología y Ciencias Experimentales; FCJE: Facultad de Ciencias Jurídicas y Económicas.

Con más exactitud, y tal y como podemos observar en la tabla 6 , las facetas en las que se han encontrado diferencias significativas son: Sentimientos $(p<0.05)$ y Valores $(p<0.005)$ 
del factor Apertura y en las facetas Franqueza $(p<0.05)$ y Modestia $(p<0.05)$ del factor Amabilidad. La faceta Sentimientos, implica la receptividad a sus propias emociones interiores y a considerar la emoción como una parte importante de la vida; Valores, indica disposición para cuestionar los valores sociales, políticos y religiosos; Franqueza, las personas que puntúan alto son francas, sinceras e ingenuas y Modestia, la persona es humilde y trata de pasar desapercibida.

En el resto de de dimensiones y facetas que analiza el NEO-PI-R no se hallaron diferencias significativas entre los estudiantes de las diferentes facultades de la Universidad.

\section{Discusión y conclusiones}

En referencia a los resultados en general de los estudiantes universitarios se observa que la muestra evaluada presenta un perfil caracterizado por la inestabilidad emocional (altas puntuaciones en Neuroticismo) y la baja amabilidad y responsabilidad. Destacamos también la baja puntuación obtenida en la faceta Asertividad. Estos resultados confirman los obtenidos por otros investigadores como Furuwaka y Shibayama $(1993,1994)$ que encontraron que el factor Neuroticismo era un predictor significativo de mal ajuste en un grupo de estudiantes japoneses que cursaban sus estudios en Estados Unidos, e incluso, desde otros estudios relacionados, se apunta a la influencia de este factor de Neuroticismo en mayores niveles de evitación social (Chataway y Berry, 1989). En este sentido, Mak y Tran (2001), en su trabajo con estudiantes vietnamitas emigrantes en Australia indican que, la importancia para la eficacia en la interacción social entre los estudiantes inmigrantes y los autóctonos no solo radicaba en la existencia de factores culturales de reubicación o adaptación a la nueva cultura, sino que además, la posesión de recursos personales tales como la eficacia social, la Extraversión y la Apertura facilitan su aculturación al nuevo contexto.

Nuestros resultados indican que es necesario dotar de recursos personales relacionados con el aumento de la autoestima, la asertividad y las estrategias de regulación emocional a los estudiantes universitarios. El conocimiento y entrenamiento en estas técnicas psicológicas influiría positivamente en el equilibrio emocional del estudiantado aumentando sus competencias personales, lo cual revertirá en una mayor competencia social y laboral futura. La competitividad del mundo laboral hace necesaria la adquisición de competencias tanto personales, como sociales, en los estudiantes universitarios por lo que, el sistema académico universitario debería plantear alternativas formativas que ofrezcan este tipo de recursos a su alumnado.

Por otra parte, en referencia a la comparación de las variables de personalidad según la rama de conocimiento, Los estudiantes de la FCHS (Facultad de Ciencias Humanas y Sociales) obtienen medias mayores en los factores de personalidad: Apertura y Amabilidad. También obtienen diferencias estadísticamente significativas en las facetas de personalidad: Sentimientos, Valores, Franqueza y Modestia. Como vemos, los estudiantes que cursan estudios relacionados con las CIEncias Humanas y Sociales tienen una serie de características de personalidad relacionadas directamente con un perfil que facilita la relación con los demás. Este hecho puede considerarse lógico dado el perfil personal y académico de los estudiantes que eligen estas carreras. No obstante, la demanda laboral actual requiere que todos los estudiantes universitarios adquieran competencias sociales y ciudadanas útiles para desarrollarse en un mundo plural y globalizado.

Es importante mencionar que los resultados obtenidos por este estudio deben interpretarse con cautela debido a la muestra limitada de participantes, especialmente, cuando se realizan comparaciones entre las ramas de conocimiento. Sería importante aumentar la media de los participantes en los distintos subgrupos para garantizar la representatividad de los datos. Aumentando la muestra, también podríamos realizar análisis entre los estudiantes de 
segundo y tercer ciclo y también en función del género. Otra cuestión interesante a tener en cuenta en futuros estudios sería el utilizar alguna medida de personalidad adicional basada en otro modelo teórico, así como otras medidas psicológicas que pudieran darnos más información sobre las habilidades, competencias y recursos psicológicos de los que disponen los estudiantes universitarios para enfrentarse a los retos del día a día. Para acabar, sería interesante utilizar un método de evaluación más adaptado a las características y modo de funcionamiento de los jóvenes universitarios, consideramos que en futuros estudios en esta línea de investigación, deberían proponerse aplicaciones on line para la recogida de los datos asegurando las cuestiones de confidencialidad y protección de datos inherentes a la investigación con humanos. Esta mejora podría suponer el acceso a más participantes.

A pesar de las limitaciones de este estudio prelimiar, sus resultados ponen de manifiesto que, posiblemente sean necesarios mayores esfuerzos desde la educación superior para garantizar los objetivos del Plan de Bolonia relacionadas con la competencia social de los estudiantes universitarios en relación con su inserción laboral en un mundo globalizado.

\section{Referencias bibliográficas}

Chataway, C. J. \& Berry, J. W. (1989). Acculturation experiences, appraisal, coping, and adaptation: A comparison of Hong Kong Chinese, French, and English students in Canada. Canadian Journal of Behavioral Science, 21, 295-309.

Costa, P. T. \& McCrae, R. R. (1992). Professional manual: Revised NEO Personality Inventory (NEO-PI-R) and NEO Five-Factor Inventory (NEO-FFI). Odessa, FL: Psychological Assessment Resources. Versión española, Cordero, A., Pamos, A. \& Seisdedos, N. (1999). Madrid: TEA Ediciones.

Cui, G. \& Awa, N. E. (1992). Measuring intercultural effectiveness: An integrative approach. International Journal of Intercultural Relations, 16, 311-328.

European Ministers Risponsable for Higher Education (2007). Comunicado de Londres. Hacia el Espacio Europeo de Educación Superior: respondiendo a los retos de un mundo globalizado. Bologna Process. European Comission: London.

European Ministers Risponsable for Higher Education (2009). The Bologna Process 2020 -The European Higher Education Area in the new decade. Bologna Process. European Comission: Lauven.

Furukawa, T. \& Shibayama, T. (1993). Predicting maladjustment of exchange students in different cultures: A prospective study. Social Psychiatry and Psychiatric Epidemiology, 28, 142-146.

Furukawa, T. \& Shibayama, T. (1994). Factors in uencing adjustment of high school students in an inernational exchange program. Journal of Nervous and Mental Disease, 182, 709-714.

Kim, Y. Y. (1988). Communcation and cross-cultural adaptation: An integrative theory. Clevedon, England:Multicultural Matters.

Mak, A. S. \& Tran, C. (2001). Big five personality and cultural relocation factors in Vietnamese Australian students' intercultural social self-efficacy. International Journal of Intercultural Relations, 25 (2): 181-201.

Rodríguez-Izquierdo, R. M. (2008). Implicaciones para los planes de estudio de la competencia intercultural en la formación de profesores principiantes. I Congreso Internacional sobre Profesorado Principiante e Inserción Profesional a la Docencia. Sevilla.

Searle, W. \& Ward, C. (1990). The prediction of psychological and sociocultural adjustment during cross-cultural transitions. International Journal of Intercultural Relations, 14, 449-464.

Ward, C. \& Kennedy, A. (1993). Where's the "culture" in cross-cultural transition? Comparative studiesof sojourner adjustment. Journal of Cross-Cultural Psychology, 24, 221-249.

Ward, C. (1996). Acculturation. In D. Landis, \& R. S. Bhagat (eds.), Handbook of Intercultural Training (pp. 124-147). Thousand Oaks, CA: Sage. 